\title{
Practical Accessibility in eLearning: Decisions, Dilemmas and Discussions for Educators and Developers
}

\author{
Martyn Cooper, Chetz Colwell, Mary Taylor \\ Institute of Educational Technology, The Open University, Walton Hall, Milton Keynes, MK7 6AA, UK \\ M.Cooper@open.ac.uk; C.Colwell@open.ac.uk; M.E.Taylor@open.ac.uk
}

\begin{abstract}
This workshop reflects on the work of the Accessibility in Educational Media group at the Open University (UK) in supporting those developing courses (academics, managers and technical developers) in making them accessible to disabled students. Through a mixture of presentations, small group activities and plenary discussion, this workshop will explore the principles and processes involved in development of accessible eLearning. There will be an emphasis on exchanging expertise and learning from each others' good practice.
\end{abstract}

Disabled Students; Accessibility; Learning Objectives; e-Learning.

\section{CONTEXT FOR WORKSHOP}

Access to online learning for disabled students has become a significant issue for all higher education establishments in the UK, particularly given the increased use of computers in the delivery of education and antidiscrimination legislation introduced in 2001. This workshop reflects on some of the issues raised in responding to this agenda from the experience at the OU. It builds its discussion around the case of online learning, that is the use of internet/intranet technologies in delivering education but the issues are largely the same for the use of educational DVDs/CD-ROMs or other computer software as part of a course. What is more, the issues can generally be extended by analogy beyond the educational sector to anyone seeking to deliver services or trade on the World Wide Web. The OU is Europe's largest educational establishment with the vast majority of its courses being delivered predominantly at a distance. The OU has invested $£ 30$ million in e-learning over recent years.

The OU currently has over 10,000 disabled students approximately half of whom receive some particular support from the university to enable them to participate in their studies. The university is committed to making its online educational content and student services accessible to disabled students but the scale of this presents a challenge. This workshop concentrates on the pedagogic, organisational and cultural issues rather than the technical ones; it argues for the importance of addressing these to effect the organisational change needed to ensure that the accessibility challenges are effectively met.

So often in accessibility considerations of educational web sites or software, the focus is on how best to make a particular element technically accessible to disabled students. However the authors maintain that educators need to stand back from these considerations and remember that fundamentally what we are seeking to make accessible is the learning. This may seem an obvious statement but it is often missed and is key to deciding what is the most appropriate response to meet the needs of disabled students. Thus fundamental to accessibility considerations in online education is having clearly established learning objectives. This is important through different degrees of granularity from the overall learning objectives for the course through to the learning objectives for the individual activities being mediated via computer.

The issue of responsibility for accessibility is often helpfully thought of in the same way that responsibility in health and safety legislation is considered. The ultimate responsibility lies with the governing body of the institution. This responsibility is devolved by them to specific named individuals or posts across the organisation, however all employees have a responsibility for health and safety. So in the case of accessibility to online learning, all involved in its conception, implementation and delivery have a responsibility for its accessibility, however specific responsibilities should be given to key people. All course team decisions on accessibility and reasonable adjustments should be documented and a protocol has been established for doing this at the OU within the formal course development process. It is important to record the rationale for these decisions, both as part of the internal 
management of course development and because it is these decisions that could be subject to legal challenge under Disability Discrimination Act Part 4 [1]. Further it is important to inform disabled students ahead of their enrolment on a course of any particular accessibility issues that elements of the course may present them and where they may be offered an alternative activity. An Accessibility Primer has also been developed to support OU staff responsible for course production [2].

It is also important to clearly establish the division of responsibilities between the educators devising the course and the developers implementing its software and web based components. Because of the fact that accessibility is intrinsically linked to the learning objectives, the approach taken at the $\mathrm{OU}$ is that it is the educators' responsibility to specify the accessibility criteria but the developers' responsibility to determine how these are met in the technology they employ, by understanding the principles underlying the accessibility guidelines available for different technologies, such as the Web Accessibility Initiative's Web Content Accessibility Guidelines [3], IBM's Guidelines for Writing Accessible Applications Using 100\% Pure Java [4], and WebAIM's Creating Accessible Flash [5].

\section{SUMMARY OF WORKSHOP'S GOALS AND ISSUES}

Through a mixture of presentations, small group activities and plenary discussion, this workshop will explore the principles and processes involved in development of accessible eLearning, including:

- $\quad$ General principles for making eLearning accessible to disabled students.

- The importance of learning objectives and their key relationship with accessibility

- The roles and responsibilities of different staff groups in the development of accessible eLearning, including educators, developers and support staff.

\section{REFERENCES}

[1] Disability Discrimination Act 1995: http://www.legislation.hmso.gov.uk/acts/acts1995/1995050.htm

[2] AEM's Accessibility Primer: http://kn.open.ac.uk/public/index.cfm?wpid=2488

[3] Web Content Accessibility Guidelines: http://www.w3.org/TR/WAI-WEBCONTENT-TECHS/\#ref-WCAG10

[4] Guidelines for Writing Accessible Applications Using 100\% Pure Java:

http://www-3.ibm.com/able/snsjavag.html

[5] Creating Accessible Flash: http://www.webaim.org/techniques/flash/ 